\begin{tabular}{|c|c|}
\hline Title & General ized Simple Theory for Estimating Lateral Leakage Loss Behavior in Silicon-on-Insul ator Ridge W aveguides \\
\hline Author(s) & Kakihara, Kuniaki; Saitoh, Kunimasa; Koshiba, Masanori \\
\hline Citation & $\begin{array}{l}\text { Journal of Lightwave Technology, 27(23), 5492-5499 } \\
\text { https://doi.org/10.1109/JLT .2009.2027720 }\end{array}$ \\
\hline Issue Date & 2009-12-01 \\
\hline Doc URL & http:/hdl.handle.net/2115/39952 \\
\hline Rights & $\begin{array}{l}\text { () } 2009 \text { IEEE. Personal use of this material is permitted. However, permission to reprint/republish this material for } \\
\text { advertising or promotional purposes or for creating new collective works for resale or redistribution to servers or lists, } \\
\text { or to reuse any copyrighted component of this work in other works must be obtained from the IEEE. }\end{array}$ \\
\hline Type & article \\
\hline File Information & JLT27-23_p5492-5499.pdf \\
\hline
\end{tabular}

Instructions for use 


\title{
Generalized Simple Theory for Estimating Lateral Leakage Loss Behavior in Silicon-on-Insulator Ridge Waveguides
}

\author{
Kuniaki Kakihara, Kunimasa Saitoh, Member, IEEE, and Masanori Koshiba, Fellow, IEEE
}

\begin{abstract}
In this paper, we numerically investigate the lateral leakage loss behavior for TM-like modes in silicon-on-insulator ridge waveguides. If the waveguide width is set to satisfy a certain condition, the leakage loss of the TM-like mode leads to be a minimum. When utilizing TM-like modes in the ridge waveguides, it is the main drawback that the tolerance for the variations in the waveguide width and operation wavelength is too small. In this paper, we propose a novel ridge waveguide structure, where a dimple is introduced at the ridge region. It is shown from the finite-element analysis that the ridge waveguide with a dimple is both low loss and fabrication tolerant.
\end{abstract}

Index Terms-Finite-element method (FEM), leaky waves, optical losses, optical waveguides, silicon-on-insulator (SOI) technology.

\section{INTRODUCTION}

$\mathbf{H}$ IGH-INDEX-CONTRAST (HIC) waveguides, which are usually fabricated on a silicon-on-insulator (SOI) wafer, have been under intensive study for the past several years since they offer a number of unique and useful properties not achievable in standard silica-based waveguides. HIC waveguides fall into three categories, depending on their core profile. The first one, a photonic wire waveguide [1]-[3], is usually formed by a silicon core surrounded by a silica cladding, and can have tight optical confinement due to the large refractive index contrast between the core and the cladding. It is therefore possible to reduce the bending radii to several micrometers, leading to ultra-small optical ICs. On the other hand, the second and the third ones, in which the etch depth does not penetrate the waveguide core, are called rib waveguide [4]-[6] and ridge waveguide [7]-[13], respectively. These waveguides are expected to be applied to voltage-controlled active optical devices such as modulators and light-emitting sources, since it is easy to impose an electric field across guided-mode region. In this paper, if the ratio of the silicon thickness in the core region $t_{1}$ to the silicon thickness in the cladding region $t_{2}, T=t_{2} / t_{1}(0<T<1)$, is relatively small, we call this structure rib waveguide. If the thickness ratio

Manuscript received October 21, 2008; revised May 06, 2009. First published September 04, 2009; current version published October 16, 2009.

The authors are with the Division of Media and Network Technologies, Graduate School of Information Science and Technology, Hokkaido University, Sapporo 060-0814, Japan (e-mail: kakihara@icp.ist.hokudai.ac.jp; ksaitoh@ist.hokudai.ac.jp; koshiba@ist.hokudai.ac.jp).

Digital Object Identifier 10.1109/JLT.2009.2027720

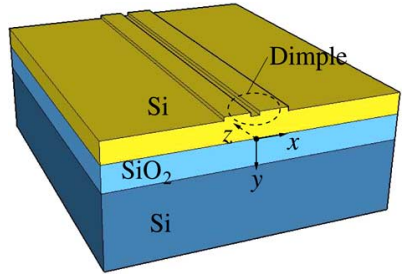

Fig. 1. Schematic representation of the SOI ridge waveguide with a dimple at the ridge region with a bird's-eye view.

$T$ is almost 1 , we call this structure ridge waveguide. In the case of $T=0,1$, they are, respectively, corresponding to a photonic wire waveguide and a slab (2-D) waveguide, which does not confine the light in the lateral direction. In particular, by employing the ridge structure, the scattering losses related to the lateral surface roughness can be reduced due to their low ridge height in the current fabrication technique. Recently, several kinds of optical devices based on SOI ridge waveguides have been reported [11]-[13].

SOI ridge waveguides possess these attractive characteristics; however, it is known that TM-like mode in ridge waveguides intrinsically leaks in the lateral cladding region for practical waveguide geometries, yet, this lateral leakage loss of the TM-like mode is minimized for particular waveguide width [10], [14], [15]. The lateral leakage loss dependence on the waveguide width for the TM-like mode in the SOI ridge waveguides has been observed experimentally [10], and it has shown that the tolerance to the variation in wavelength and waveguide width is very low. Therefore, it is necessary to improve these leakage loss properties to utilize the TM-like mode. Recently, to improve the leakage loss properties, a ridge waveguide structure, where a dimple is introduced at the ridge center as shown in Fig. 1, has been proposed [16]. However, the dimple position is only limited to the center of the ridge region.

The purpose of this paper is to discuss the lateral leakage loss characteristics for the TM-like mode in SOI ridge waveguides. We derive a simple theory for estimating lateral leakage loss behavior in SOI ridge waveguides. It is shown from finite-element-based [17] and effective-index-based analyses that SOI ridge waveguides with low-loss operation over a wide wavelength range and good fabrication tolerance could be designed for TM-like modes. And we discuss the application of this design procedure to ridge-type horizontal slot waveguides [18]-[20]. 


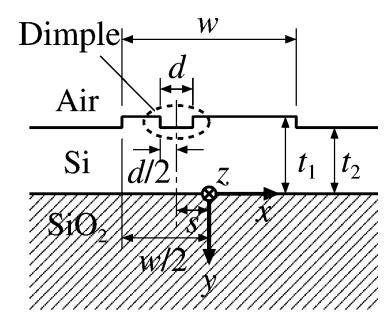

(a)

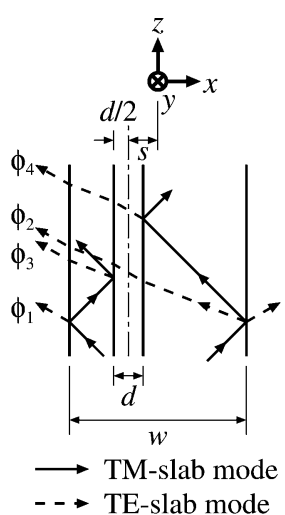

(b)
Fig. 2. (a) Cross section of the SOI ridge waveguide with a dimple at the ridge region and (b) propagation process of the TM-like mode in a ridge waveguide with a top view, where the main field components of the TM-like hybrid mode are the $y$ component of electric fields $E_{y}$ and the $x$ component of magnetic fields $H_{x}$, and the TE- and TM-slab modes (intrinsically propagating in 2-D, planar waveguides) have no $y$ component of electric and magnetic fields, $E_{y}$ and $H_{y}$, respectively.

\section{THEORY FOR ESTIMATING LATERAL LEAKAGE LOSSES}

We consider an SOI ridge waveguide with a dimple at the ridge region, as shown in Fig. 2(a), where the refractive indexes of the core and the cladding are taken as $n_{\text {core }}=3.475$ and $n_{\text {clad }}=1.444$, respectively, assuming $\mathrm{Si}$ and $\mathrm{SiO}_{2}$. The structural parameters, $w, d, t_{1}, t_{2}$, and $s$, represent the waveguide width, the dimple width, the central core thickness, the lateral slab cladding thickness, and the distance between the ridge center and the dimple center, respectively.

The ridge waveguides can be assumed to be perturbation of the planar waveguides, and the modes in the ridge waveguides are hybrid in nature, namely TE-like or TM-like hybrid mode. In the planar waveguides, the transverse field component of the TM-slab/TE-slab mode is only $E_{y} / E_{x}$. In the ridge waveguides, the main transverse field component of the TM-like mode is the $E_{y}$ (TM-slab component), and the minor transverse field component is $E_{x}$ (TE-slab component).

The lateral leakage phenomenon in ridge waveguides is explained by the mode conversion between TE- and TM-slab modes at the ridge boundaries and an effective index method [10], [14], [15]. In this method, we regard an effective refractive index of the guided mode in the slab (2-D) waveguide as a refractive index of the lateral cladding region. Fig. 3 shows the effective refractive index of the TE and TM modes in the slab waveguide as a function of the slab thickness.

We consider a case that a TM-slab wave is incident on the ridge boundary, as shown in Fig. 2(b). If the lateral cladding thickness $t_{2}$ is zero or small enough, the TM-like mode is confined to the core region without leakage loss, since the effective index of the TM-like mode becomes larger than that of not only the TM-slab, but also the TE-slab mode in the lateral cladding. In this case, both the TM-slab wave and the TE-slab wave in the cladding region are always evanescent. On the other hand, if $t_{2}$ is large enough, lateral leakage of the TM-like mode occurs because the effective index of the TM-like mode becomes smaller than that of the TE-slab mode. In this case, only the TM-slab

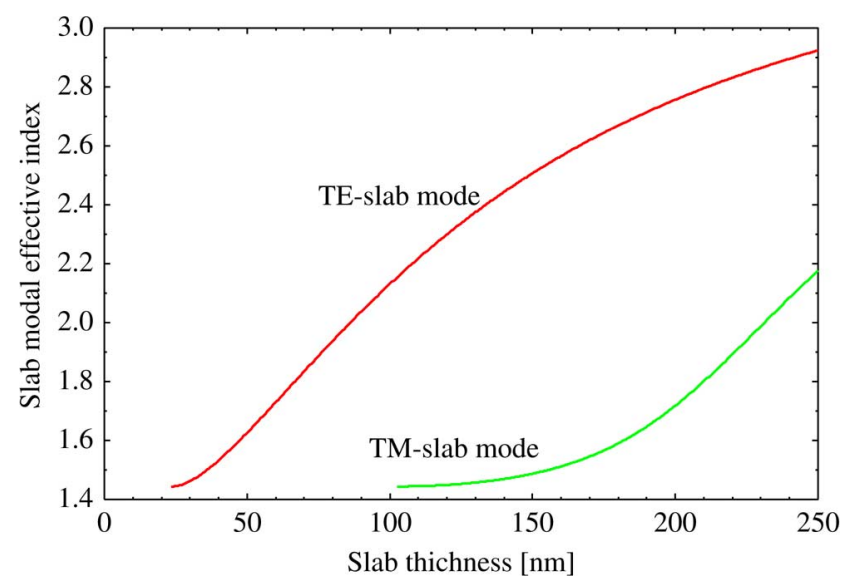

Fig. 3. Effective refractive index of the TE and TM modes in the slab waveguide as a function of the slab thickness.

wave is evanescent and the TE-slab wave radiates in the lateral direction, as shown in Fig. 2(b).

At the ridge and dimple boundaries, the mode conversion from the TM-slab wave to the transmitted and reflected TE-slab waves can be occurred. Suppose that the transmitted and reflected TE-slab waves [dashed line as shown in Fig. 2(b)] are approximately equal in magnitude $\phi_{0}$ with a relative phase difference of $\pi$ [10], [14], [15], which is also described in $\left|\theta_{t}-\theta_{r}\right|=$ $\pi$. All the transmitted and reflected TE-slab waves at the step discontinuities, as shown in Fig. 2(b), are expressed as follows:

$$
\begin{aligned}
& \phi_{1}=\phi_{0} \exp \left(j \theta_{t}\right) \\
& \phi_{2}=\phi_{0} \exp \left[-j\left\{k_{1}(w-d)+k_{2} d\right\}\right] \exp \left(j \theta_{r}\right) \\
& \phi_{3}=\phi_{0} \exp \left(-j k_{1} \frac{w-d-2 s}{2}\right) \exp \left(j \theta_{r}\right) \\
& \phi_{4}=\phi_{0} \exp \left\{-j\left(k_{1} \frac{w-d-2 s}{2}+k_{2} d\right)\right\} \exp \left(j \theta_{t}\right)
\end{aligned}
$$

with

$$
\begin{aligned}
& k_{1}=k_{0} \sqrt{n_{\mathrm{TE}}^{2}\left(t_{1}\right)-N_{\mathrm{eff}, \mathrm{TM}}^{2}} \\
& k_{2}=k_{0} \sqrt{n_{\mathrm{TE}}^{2}\left(t_{2}\right)-N_{\mathrm{eff}, \mathrm{TM}}^{2}}
\end{aligned}
$$

where $k_{0}$ is the wavenumber in vacuum, $N_{\text {eff,TM }}$ is the effective refractive index of the TM-like mode in the ridge waveguide, and $n_{\mathrm{TE}}\left(t_{i}\right)(i=1$ or 2$)$ is the effective refractive index of the TE-slab mode in the planar waveguide with thickness $t_{i}$. The total optical intensity of the transmitted TE-slab wave is approximately expressed as interference between the TE-slab waves generated at the four-step discontinuities

$$
\begin{aligned}
I \propto & \left|\phi_{1}+\phi_{2}+\phi_{3}+\phi_{4}\right|^{2} \\
= & 4\left|\phi_{0}\right|^{2}\left[\left\{1-\cos \frac{k_{1}(w-d)}{2}\right\}\right. \\
& \times\left\{1+\cos \frac{k_{1}(w-d)+2 k_{2} d}{2}\right\} \\
& \left.+2\left(1-\cos k_{1} s\right) \sin \left\{k_{1}(w-d)+2 k_{2} d\right\} \sin \left(k_{2} d\right)\right]
\end{aligned}
$$




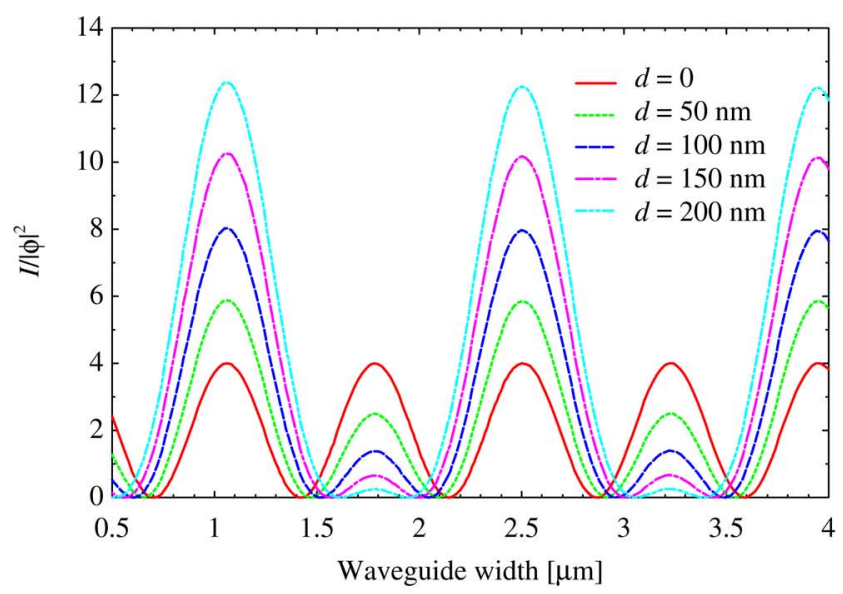

(a)

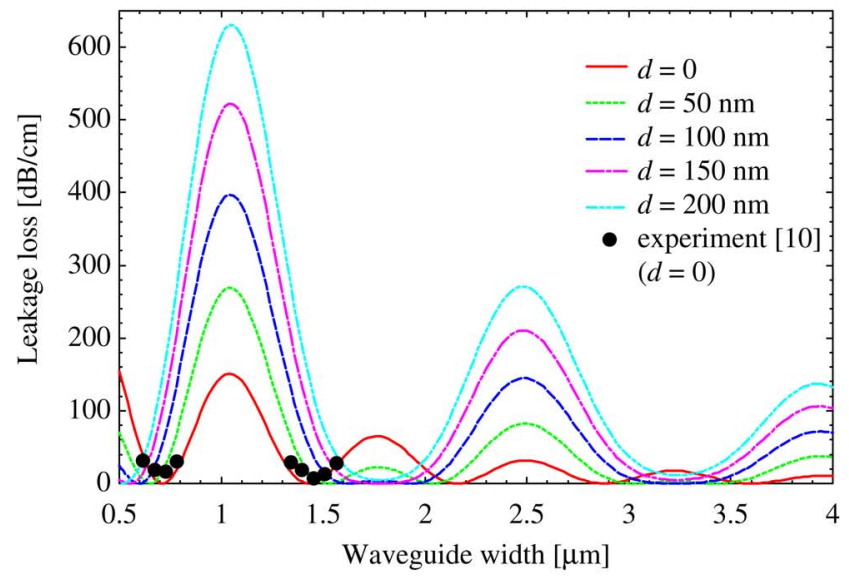

(b)

Fig. 4. Waveguide width dependence of: (a) the normalized intensity $I$ of the transmitted TE-slab waves obtained from (4) and (b) the leakage losses of the TM-like mode obtained from FEM simulation in the ridge waveguide in Fig. 2(a), where the dimple position is fixed as $s=0$ and the dimple width $d$ is taken as a parameter.

If $s=0$, the following simple equation is derived:

$$
\begin{aligned}
I \propto 4\left|\phi_{0}\right|^{2}\left[1-\cos \frac{k_{1}(w-d)}{2}\right] & \\
& \times\left[1+\cos \frac{k_{1}(w-d)+2 k_{2} d}{2}\right] .
\end{aligned}
$$

To evaluate the leakage losses of the TM-like mode in SOI ridge waveguides and the normalized intensity $I$ of the transmitted TE-slab waves, a full vectorial numerical approach is necessary. In this study, the vector finite-element method (FEM) with perfectly matched layer [17] is used.

\section{NUMERICAL RESULTS}

Fig. 4(a) and (b) shows, respectively, the normalized intensity $I$ of the transmitted TE-slab waves calculated from (4) and the leakage losses of the TM-like mode calculated by using the FEM as a function of the waveguide width for several incremental values of the dimple width $d$, where the operating wavelength is $\lambda=1.55 \mu \mathrm{m}$, the central core thickness is $t_{1}=205 \mathrm{~nm}$, and the lateral slab cladding thickness is $t_{2}=190 \mathrm{~nm}$ [10]. We can clearly see that the waveguide width for the minimum/maximum intensity $I$ of the transmitted

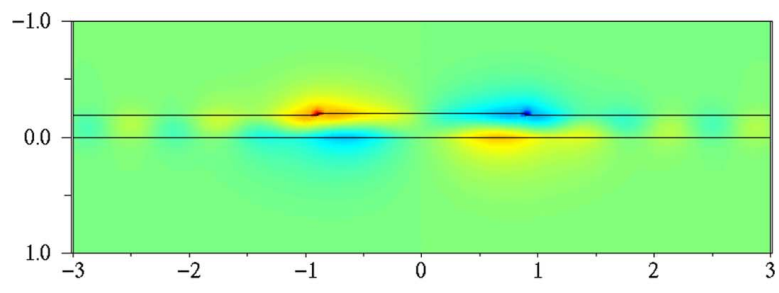

(a)

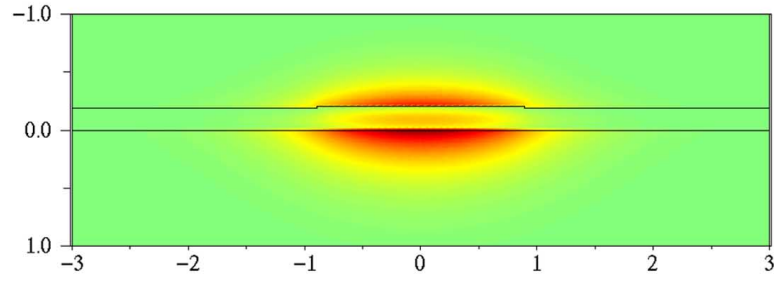

(b)

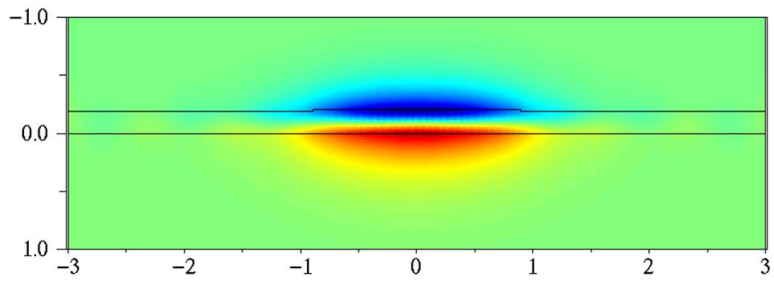

(c)

Fig. 5. (a) $x$ component, (b) $y$ component, and (c) $z$-component of the electric field distributions of the TM-like mode in the conventional $(d=0)$ ridge waveguide.

TE-slab waves corresponds to that for the minimum/maximum leakage losses of the TM-like mode. Therefore, we can easily estimate the waveguide width corresponding to the minimum/maximum leakage loss of the TM-like mode from (4).

For the conventional ridge waveguide structure $(d=0),(4)$ is reduced to

$$
I \propto 2\left|\phi_{0}\right|^{2}\left(1-\cos k_{1} w\right)
$$

and thus, the waveguide width that makes leakage loss minimum/maximum is estimated from the relation $k_{1} w=m \pi$ [10], [14], [15], where $m$ is a positive integer. If $m$ is even (or odd), the leakage loss becomes minimum (or maximum). The leakage loss behavior of the conventional structure $(d=0)$ obtained by the FEM simulation agrees approximately with the experimental one [10]. Figs. 5 and 6 show, respectively, the electric field distributions of the fundamental TM-like mode in the conventional ridge waveguide $(d=0)$ and the proposed ridge waveguide with $w=1765 \mathrm{~nm}(m=5)$ at the operating wavelength of $1.55 \mu \mathrm{m}$. We can clearly see that the TE-slab components $\left(E_{x}\right.$ and $\left.E_{z}\right)$ leak to the lateral cladding region for the conventional ridge structure. On the other hand, the TE-slab components do not leak to the lateral cladding region for the proposed ridge structure. In the proposed ridge waveguide structure $(d>0)$, the waveguide width that makes leakage loss minimum varies with the dimple width, while the waveguide width corresponding to the maximum leakage loss hardly depends on the dimple width variation. If the waveguide width $w$ is set to around $1783(m=5)$ and $3229 \mathrm{~nm}(m=9)$, the leakage losses 


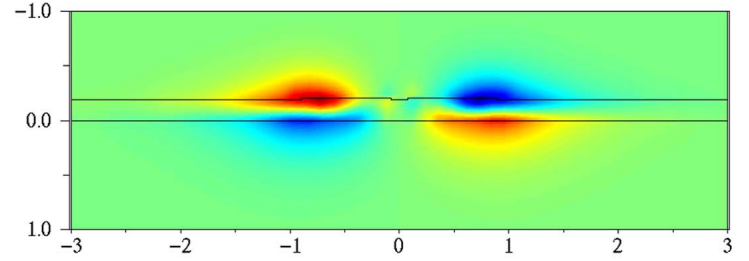

(a)

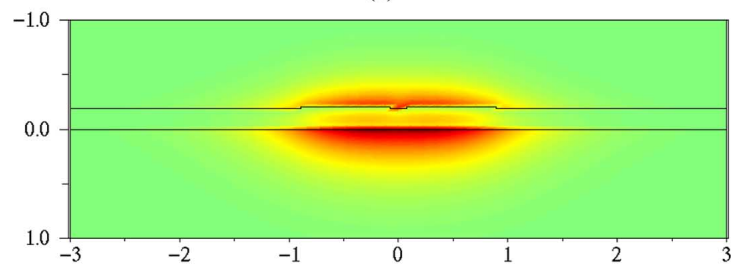

(b)

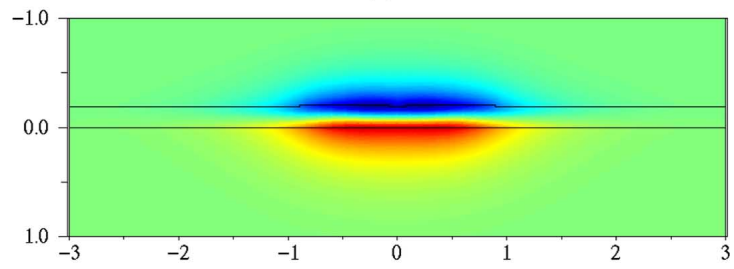

(c)

Fig. 6. (a) $x$ component, (b) $y$ component, and (c) $z$ component of the electric field distributions of the TM-like mode in the proposed ridge waveguide with a dimple.

are maximized in the case of $d=0$, whereas by increasing the dimple width, the leakage losses at these waveguide widths can be reduced, as shown in Fig. 4(b). We can clearly see that the tolerance of the waveguide width $w$ to the leakage loss can be improved by introducing the dimple at the ridge region.

Fig. 7(a) and (b) shows, respectively, the normalized intensity $I$ of the transmitted TE-slab waves calculated from (3) and the leakage losses of the TM-like mode calculated by using the FEM as a function of the waveguide width in the asymmetric waveguide structure $(s>0)$, where $\lambda=1.55 \mu \mathrm{m}$, $t_{1}=205 \mathrm{~nm}, t_{2}=190 \mathrm{~nm}$, and $d=100 \mathrm{~nm}$. We can see that the waveguide width for the minimum/maximum intensity $I$ of the transmitted TE-slab waves agrees with that for the minimum/maximum leakage losses of the TE-like mode. In the asymmetric waveguide structure $(s>0)$, the lateral leakage of the TM-like mode is not suppressed to zero.

Fig. 8 shows the leakage losses of the TM-like mode as a function of the waveguide width for several incremental values of $d$, where $\lambda=1.55 \mu \mathrm{m}, t_{1}=205 \mathrm{~nm}$, and $t_{2}=190 \mathrm{~nm}$. We can clearly see that if $d$ exceeds $146 \mathrm{~nm}$, the leakage losses do not drop to zero, and if $d$ is less than $134 \mathrm{~nm}$, the waveguide width range with the leakage loss less than $0.1 \mathrm{~dB} / \mathrm{cm}$ is divided into two sections. In the case of $d=140 \mathrm{~nm}$, low leakage loss operations less than $0.1 \mathrm{~dB} / \mathrm{cm}$ are realized for waveguide width variations from $w=1715$ to $1840 \mathrm{~nm}$. It means that the variation of the waveguide width has a small effect on the leakage losses of the TM-like modes compared with the variation of $d$.

Fig. 9(a)-(c) shows the waveguide width dependence of the leakage losses with the central core thickness $t_{1}$, the lateral

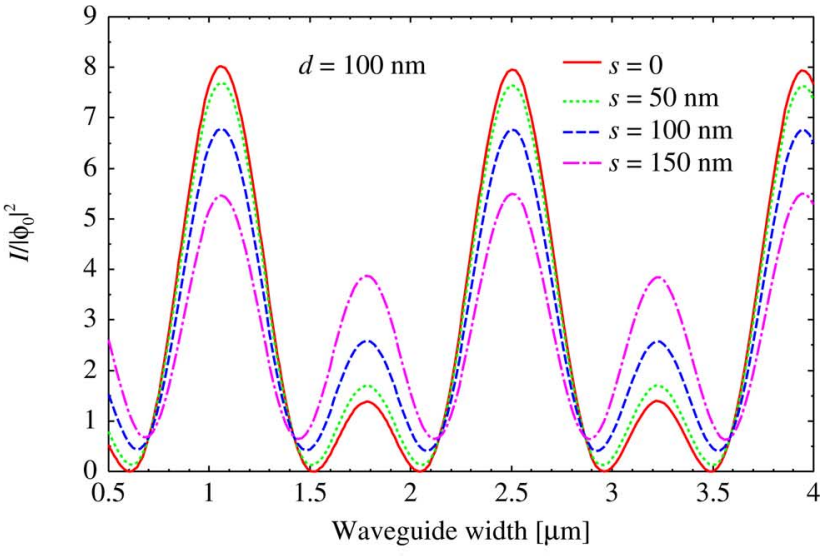

(a)

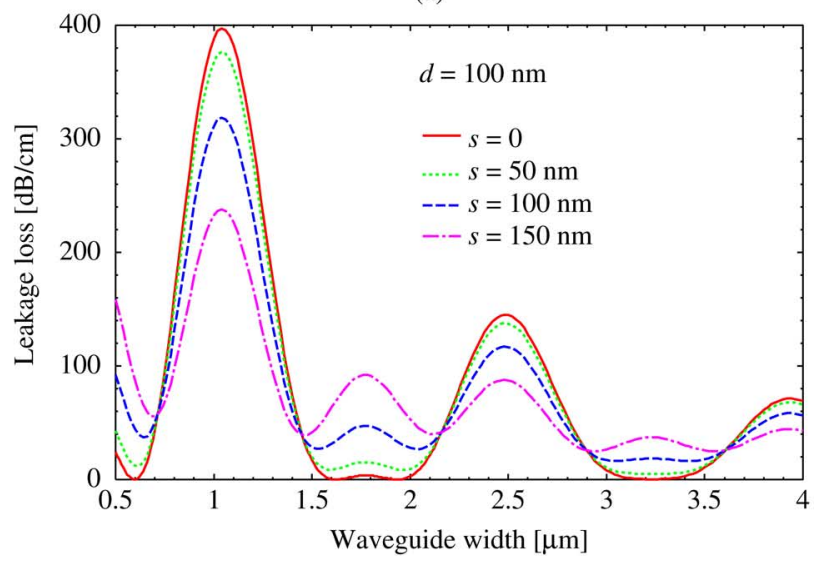

(b)

Fig. 7. Waveguide width dependence of: (a) the normalized intensity $I$ of the transmitted TE-slab waves obtained from (3) and (b) the leakage losses of the TM-like mode obtained from the FEM simulation in the ridge waveguide in Fig. 2(a), where the dimple width is fixed as $d=100 \mathrm{~nm}$ and the dimple position $s$ is taken as a parameter.

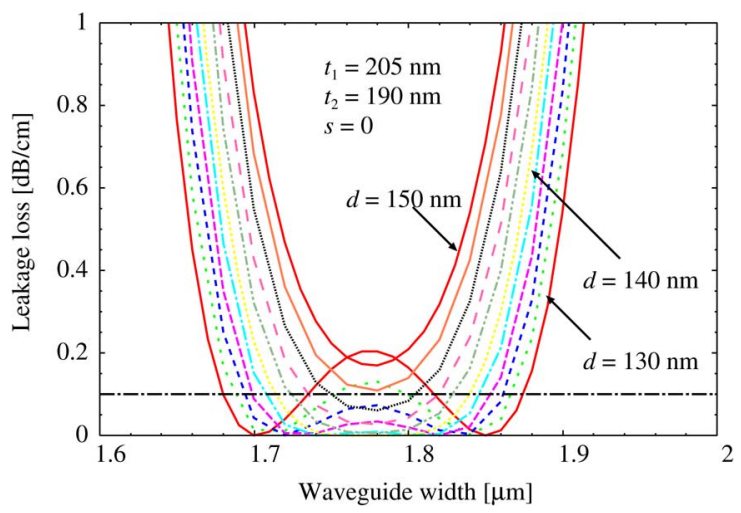

Fig. 8. Waveguide width dependence of the leakage losses with the dimple width $d$ and variations for the wavelength $\lambda=1.55 \mu \mathrm{m}$.

cladding thickness $t_{2}$, and the lateral shift of the dimple $s$ for the operating wavelength $\lambda=1.55 \mu \mathrm{m}$ and the dimple width of $d=140 \mathrm{~nm}$. The leakage losses are very sensitive to the variation of these structural parameters; therefore, these waveguides 


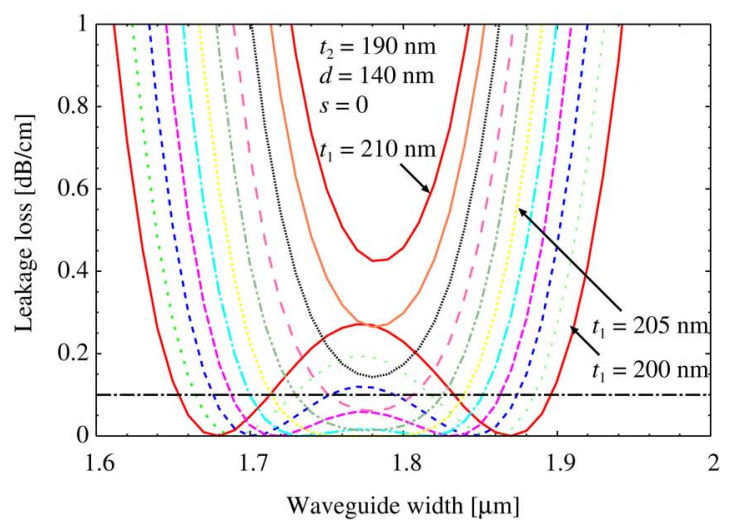

(a)

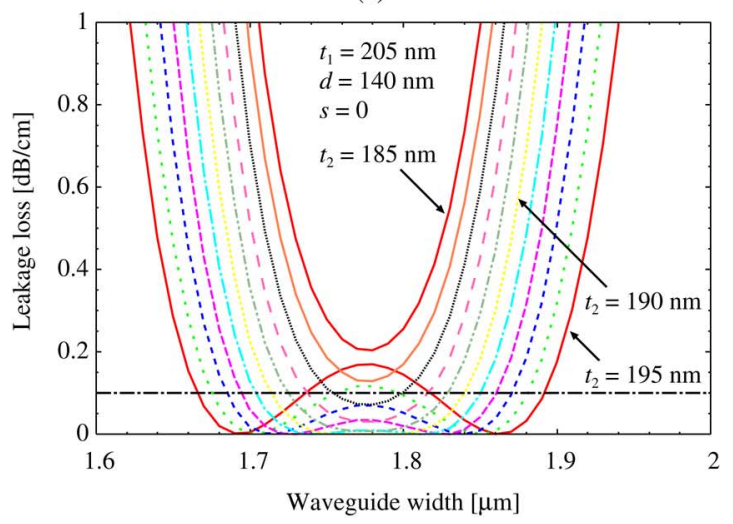

(b)

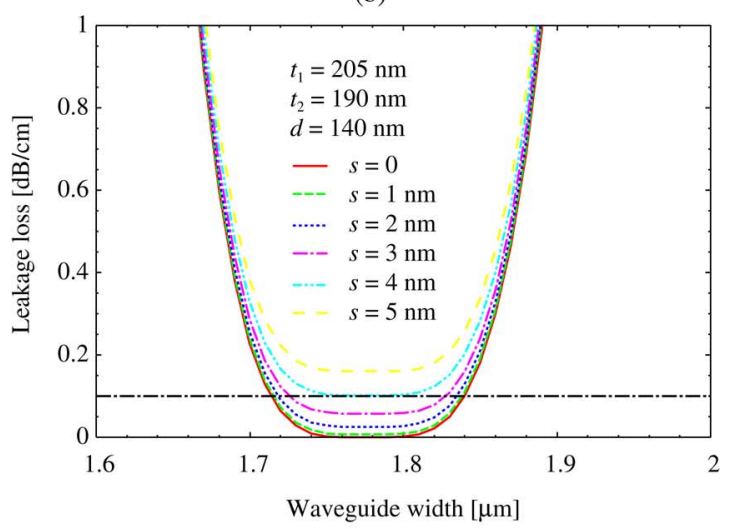

(c)

Fig. 9. Waveguide width dependence of the leakage losses with: (a) the central core thickness $t_{1}$, (b) the lateral slab cladding thickness $t_{2}$, and (c) the lateral shift of the dimple $s$ variations for the dimple width $d=140 \mathrm{~nm}$ and the wavelength $\lambda=1.55 \mu \mathrm{m}$.

should be controlled by the fabrication process of nanometer order.

\section{ApPliCATION TO THE RidGE-TyPE HoRIZONTAL SLOT WAVEGUIDES}

The proposed design procedure can be applied to the ridgetype horizontal slot waveguides [18]-[21], as shown in Fig. 10. In the slot waveguide, the guided mode whose major electric field component is perpendicular to the material interface in the slot region (in this paper, polarized along the $y$-axis, namely TM-like mode) is strongly confined in the low refractive index material sandwiched by the high refractive index materials. In

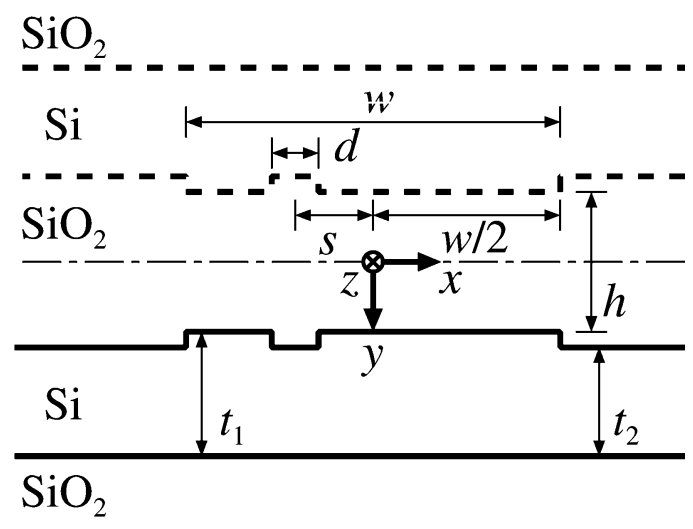

Fig. 10. Schematic representation of the ridge-type horizontal slot waveguides with a dimple at the ridge region.

order to satisfy that the electric flux density is continuous, the electric field amplitude in the low refractive index region is much higher than that in the high refractive index region. Aside from horizontal slot waveguides, vertical slot waveguides have also been investigated, and some of the optical devices were proposed such as ring resonator, directional coupler, and all-optical logic gate [22]-[24]. Compared to the vertical slot waveguide, there are some advantages to the horizontal slot waveguide. The horizontal slot structure can reduce the scattering losses related to the surface roughness since the horizontal slot surface is easier to control than the vertical one in the current fabrication technique, and consequently, it is easy to control the slot width in the order of several tens of micrometers with miniaturized surface roughness.

In a recent work [19], Muellner et al. have investigated the lateral leakage loss characteristics of the TM-like mode in the ridge-type horizontal slot waveguides. The principle of lateral leakage phenomenon in the ridge-type horizontal slot waveguide is more complex than the simple ridge waveguide. The slot waveguide is regarded as a perturbation of a five-layer slab (2-D) waveguide. These waveguides consist of coupled system of two slab/ridge waveguides, which result in two (even/odd) modes for TE- and TM-like modes, respectively. In the even mode, the major electric field components are symmetrical with respect to the $x$-axis. In the odd mode, on the other hand, the major electric field components are antisymmetrical with respect to the $x$-axis. If $t_{2} / t_{1}$ is large enough, the TM-like mode in this slot waveguide leaks in the lateral cladding region, which is explained in much the same manner as shown in the previous section. In the case of perfectly symmetric structures, the TM-like mode leak in the lateral cladding region coupled to only odd TE-slab mode because of the same parity between odd TE-slab mode and TM-like modes.

In order to improve the leakage loss properties, we consider the ridge-type horizontal slot waveguide with a dimple at the ridge region, as shown in Fig. 10. The total optical intensity of the transmitted TE-slab wave in this slot waveguide is the same as (3), but only (2a) and (2b) should be replaced with

$$
\begin{aligned}
& k_{1}=k_{0} \sqrt{n_{\mathrm{TE}}^{2}\left(t_{1}, h\right)-N_{\mathrm{eff}, \mathrm{TM}}^{2}} \\
& k_{2}=k_{0} \sqrt{n_{\mathrm{TE}}^{2}\left(t_{2}, h\right)-N_{\mathrm{eff}, \mathrm{TM}}^{2}} .
\end{aligned}
$$




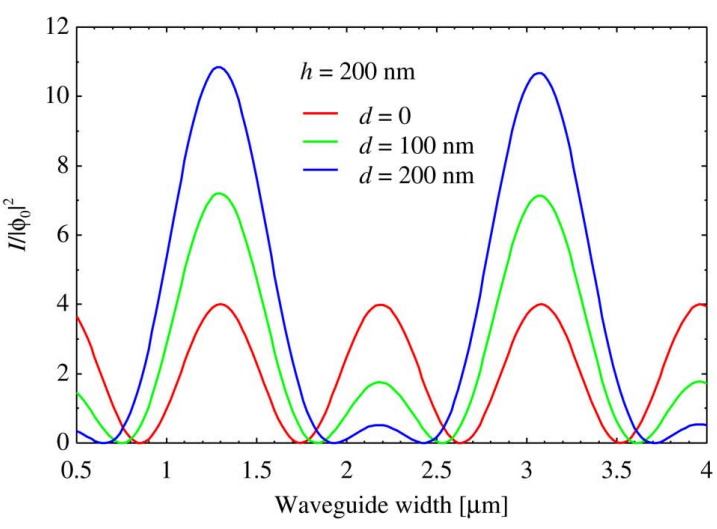

(a)

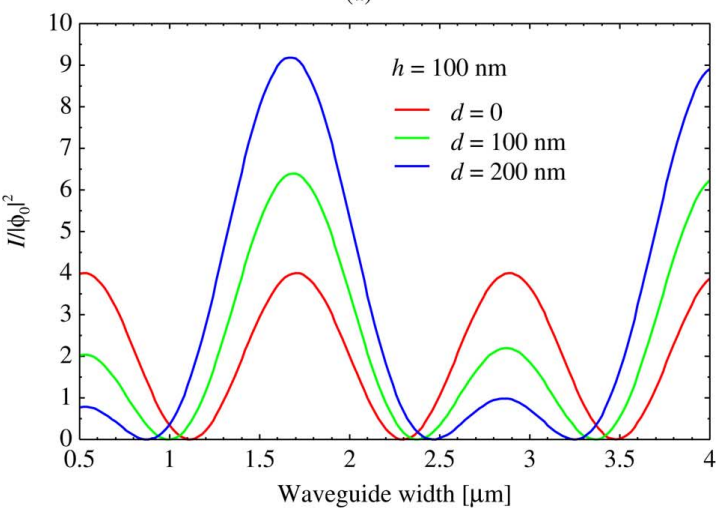

(b)

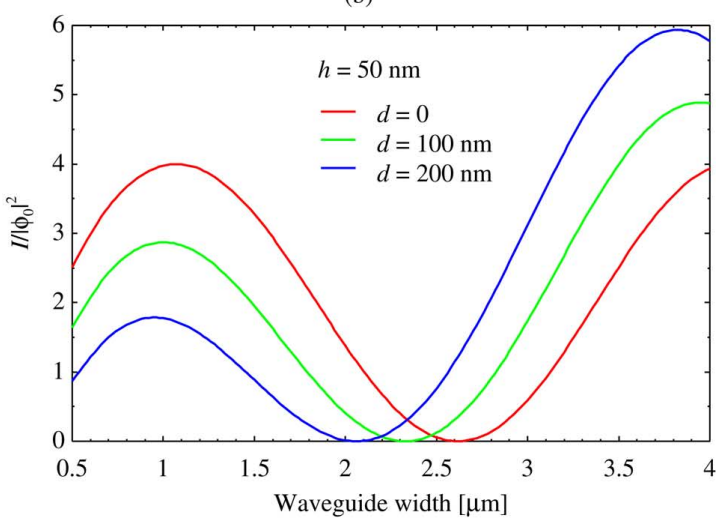

(c)

Fig. 11. Waveguide width dependence of the normalized intensity $I$ of the transmitted TE-slab waves obtained from (4) in the ridge-type horizontal slot waveguide for: (a) $h=200 \mathrm{~nm}$, (b) $h=100 \mathrm{~nm}$, and (c) $h=50 \mathrm{~nm}$.

Figs. 11 and 12 show, respectively, the normalized intensity $I$ of the transmitted TE-slab waves calculated from (3) and the leakage losses of the TM-like mode calculated by using the FEM as a function of the waveguide width for several incremental values of the dimple width $d$, where the operating wavelength is $\lambda=1.55 \mu \mathrm{m}, t_{1}=205 \mathrm{~nm}$, and $t_{2}=190 \mathrm{~nm}$ for different slot width $h$. We can clearly see that the waveguide width for the minimum/maximum intensity $I$ of the transmitted TE-slab waves corresponds to that for the minimum/ maximum leakage losses of the TM-like mode. If we set the waveguide width $w$ corresponding to $m=5$, the tolerance of the waveguide width to the leakage loss can be improved as well as the case of simple ridge waveguides. However, as the slot

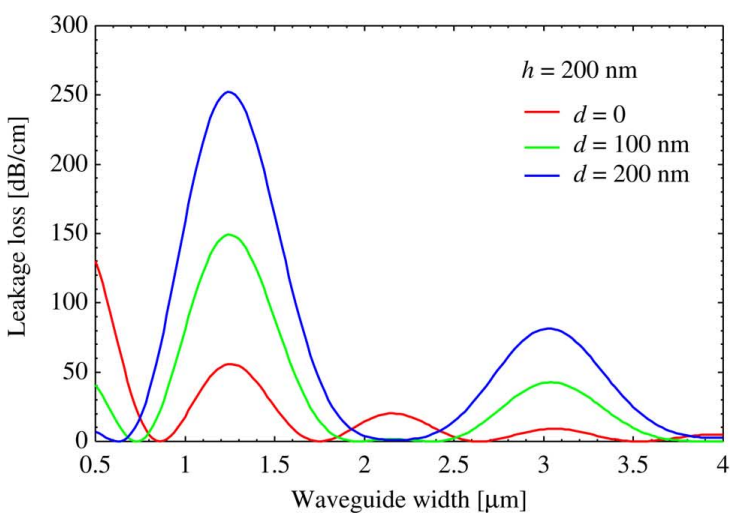

(a)

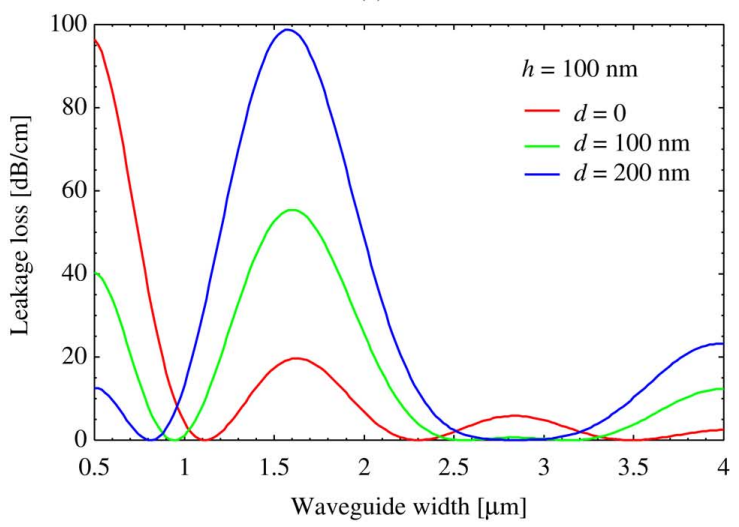

(b)

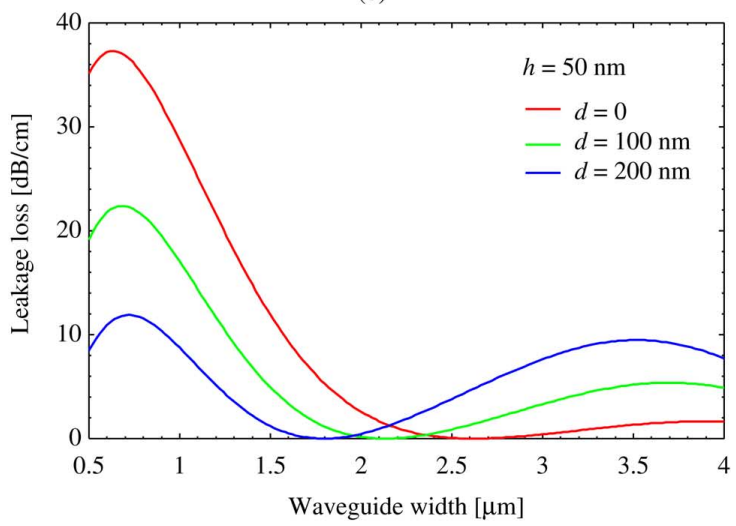

(c)

Fig. 12. Waveguide width dependence of the leakage losses of the TM-like mode obtained from FEM simulation in the ridge-type horizontal slot waveguide for: (a) $h=200 \mathrm{~nm}$, (b) $h=100 \mathrm{~nm}$, and (c) $h=50 \mathrm{~nm}$.

width $h$ becomes smaller, the difference between the width that minimizes/maximizes the leakage loss and the width that minimizes/maximizes the intensity $I$ of transmitted TE-slab waves becomes larger, which indicates the limit of application of the estimation formula for the small value of $h$.

Fig. 13(a)-(d) shows, respectively, the waveguide width dependence of the leakage losses with the dimple width $d$, the central core thickness $t_{1}$, the lateral cladding thickness $t_{2}$, and the lateral shift of the dimple $s$ for $\lambda=1.55 \mu \mathrm{m}, d=170 \mathrm{~nm}$, and $h=100 \mathrm{~nm}$. The leakage losses are very sensitive to the variation of these structural parameters; therefore, these waveguides should be controlled by the fabrication process of nanometer order. 


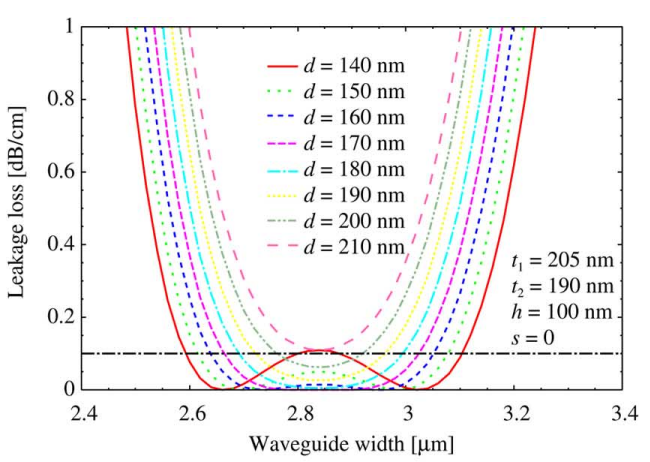

(a)

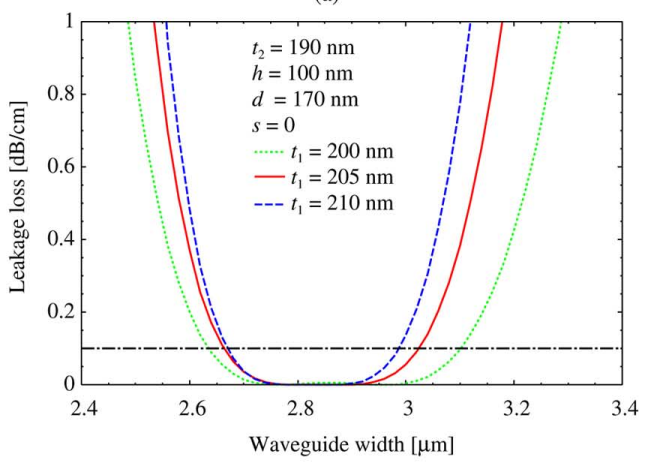

(b)

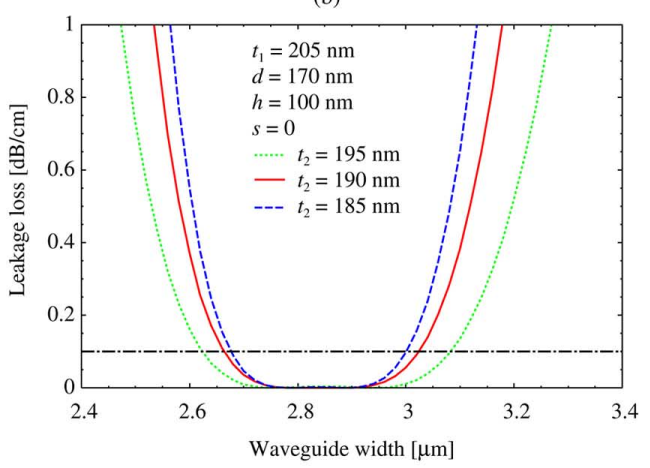

(c)

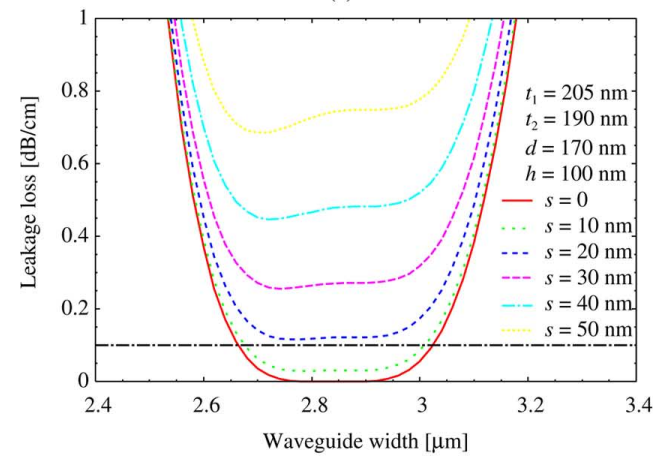

(d)

Fig. 13. Waveguide width dependence of the leakage losses with: (a) the dimple width $d$, (b) the central core thickness $t_{1}$, (c) the lateral slab cladding thickness $t_{2}$, and (d) the lateral shift of the dimple $s$ variations for the wavelength $\lambda=1.55 \mu \mathrm{m}$.

\section{CONCLUSION}

We have proposed generalized simple theory for estimating lateral leakage loss behavior in SOI ridge waveguides with a dimple at the ridge region. The theory is based on the mode conversion between TE- and TM-slab modes and the interference between the TE-slab waves generated at the ridge boundary. The comparison with losses calculated from FEM simulation supports the validity of the present approach. The presented approach can be applied to the horizontal ridge-type slot waveguide.

\section{REFERENCES}

[1] J. S. Foresi, P. R. Villeneuve, J. Ferrera, E. R. Thoen, G. Steinmeyer, S. Fan, J. D. Joannopoulos, L. C. Kimerling, H. I. Smith, and E. P. Ippen, "Photonic-bandgap microcavities in optical waveguides," Nature, vol. 390, no. 6656, pp. 143-145, Nov. 1997.

[2] F. Ohno, K. Sasaki, A. Motegi, and T. Baba, "Reduction in sidelobe level in ultracompact arrayed waveguide grating demultiplexer based on Si wire waveguide," Jpn. J. Appl. Phys., vol. 45, no. 8A, pp. 6126-6131, Aug. 2006.

[3] H. Fukuda, K. Yamada, T. Tsuchizawa, T. Watanabe, H. Shinojima, and S. Itabashi, "Silicon photonic circuit with polarization diversity," Opt. Exp., vol. 16, no. 7, pp. 4872-4880, Mar. 2008.

[4] R. A. Soref, J. Schmidtchen, and K. Petermann, "Large single-mode rib waveguides in $\mathrm{GeSi}-\mathrm{Si}$ and $\mathrm{Si}$-on-SiO ${ }_{2}$," IEEE J. Quantum Electron., vol. 27, no. 8, pp. 1971-1974, Aug. 1991.

[5] S. P. Chan, C. E. Png, S. T. Lim, G. T. Reed, and V. M. N. Passaro, "Single-mode and polarization-independent silicon-on-insulator waveguides with small cross section," J. Lightw. Technol., vol. 23, no. 6, pp. 2103-2111, Jun. 2005.

[6] M. Krause, H. Renner, and E. Brinkmeyer, "Polarization-dependent curvature loss in silicon rib waveguides," IEEE J. Sel. Topics Quantum Electron., vol. 12, no. 6, pp. 1359-1362, Nov./Dec. 2006.

[7] A. Liu, R. Jones, L. Liao, D. Samara-Rubio, D. Rubin, O. Cohen, R. Nicolaescu, and M. Paniccia, "A high-speed silicon optical modulator based on a metal-oxide-semiconductor capacitor," Nature, vol. 427, no. 6975, pp. 615-618, Feb. 2004.

[8] H. Rong, R. Jones, A. Liu, O. Cohen, D. Hak, A. Fang, and M. Paniccia, “A continuous-wave Raman silicon laser," Nature, vol. 433, no. 7027, pp. 725-728, Feb. 2005.

[9] M. A. Webster, R. M. Pafchek, G. Sukumaran, and T. L. Koch, "Low-loss quasi-planar ridge waveguides formed on thin silicon-on-insulator," Appl. Phys. Lett., vol. 87, no. 23, pp. 231108-1-231108-3, Dec. 2005.

[10] M. A. Webster, R. M. Pafchek, A. Mitchell, and T. L. Koch, "Width dependence of inherent TM-mode lateral leakage loss in silicon-oninsulator ridge waveguides," IEEE Photon. Technol. Lett., vol. 19, no. 6, pp. 429-431, Mar. 2007.

[11] R. Pafchek, R. Tummidi, J. Li, M. A. Webster, E. Chen, and T. L. Koch, "Low-loss silicon-on-insulator shallow-ridge TE and TM waveguides formed using thermal oxidation," Appl. Opt., vol. 48, no. 5, pp. 958-963, Feb. 2009.

[12] T. G. Nguyen, R. S. Tummidi, T. L. Koch, and A. Mitchell, "Lateral leakage in TM-like whispering gallery mode of thin-ridge silicon-oninsulator disk resonators," Opt. Lett., vol. 34, no. 7, pp. 980-982, Apr. 2009.

[13] T. G. Nguyen, R. S. Tummidi, T. L. Koch, and A. Mitchell, "Regorous modeling of lateral leakage loss in SOI thin-ridge waveguides and couplers," IEEE Photon. Technol. Lett., vol. 21, no. 7, pp. 486-488, Apr. 2009.

[14] A. A. Oliner, S. T. Peng, T. I. Hsu, and A. Sanchez, "Guidance and leakage properties of a class of open dielectric waveguides: Part II-New physical effects," IEEE Trans. Microw. Theory Tech., vol. 29, no. 9, pp. 855-869, Sep. 1981.

[15] K. Ogusu, "Optical strip waveguide: A detailed analysis including leaky modes," J. Opt. Soc. Amer., vol. 73, no. 3, pp. 353-357, Mar. 1983.

[16] M. Koshiba, K. Kakihara, and K. Saitoh, "Reduced lateral leakage losses of TM-like modes in silicon-on-insulator ridge waveguides," Opt. Lett., vol. 33, no. 17, pp. 2008-2010, Sep. 2008.

[17] K. Saitoh and M. Koshiba, "Full-vectorial imaginary-distance beam propagation method based on a finite element scheme: Application to photonic crystal fibers," IEEE J. Quantum Electron., vol. 38, no. 7, pp. 927-933, Jul. 2002.

[18] C. A. Barrios and M. Lipson, "Electrically driven silicon resonant light emitting device based on slot-waveguide," Opt. Exp., vol. 13, no. 25, pp. 10092-10101, Dec. 2005. 
[19] P. Muellner, N. Finger, and R. Hainberger, "Lateral leakage in symmetric SOI rib-type slot waveguides," Opt. Exp., vol. 16, no. 1, pp. 287-294, Jan. 2008.

[20] N.-N. Feng, J. Michel, and L. C. Kimerling, "Optical field concentration in low-index waveguides," IEEE J. Quantum Electron., vol. 42, no. 9, pp. 885-890, Sep. 2006.

[21] R. M. Pafchek, J. Li, R. S. Tummidi, and T. L. Koch, "Low loss $\mathrm{Si}_{-} \mathrm{SiO}_{2}$-Si 8-nm slot waveguides," IEEE Photon. Technol. Lett., vol. 21, no. 6, pp. 353-355, Mar. 2009.

[22] Q. Xu, V. R. Almeida, R. R. Panepucci, and M. Lipson, "Experimental demonstration of guiding and confining light in nanometer-size lowrefractive-index material," Opt. Lett., vol. 29, no. 14, pp. 1626-1628, Jul. 2004.

[23] T. Fujisawa and M. Koshiba, "Polarization-independent optical directional coupler based on slot waveguides," Opt. Lett., vol. 31, no. 1, pp. 56-58, Jan. 2006

[24] T. Fujisawa and M. Koshiba, "All-optical logic gates based on nonlinear slot-waveguide couplers," J. Opt. Soc. Amer. B, Opt. Phys., vol. 23, no. 4, pp. 684-691, Apr. 2006.

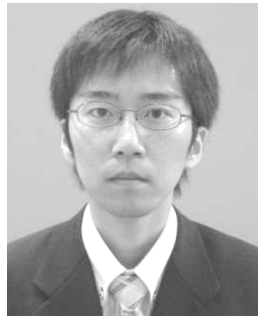

Kuniaki Kakihara was born in Furano, Japan. He received the B.S. and M.S. degrees in electronic engineering in 2006 and 2008, respectively, from the Graduate School of Information Science and Technology, Hokkaido University, Sapporo, Japan, where he is currently working toward the Ph.D. degree in the Division of Media and Network Technologies.

His current research interests include research on wave electronics, integrated optical devices, and computer-aided design and modeling of guided-wave devices using the finite-element method and beam propagation method. His work was partly funded by Japan Society for the Promotion of Science Research Fellowships for Young Scientists.

Mr. Kakihara is a Student Member of the Institute of Electronics, Information and Communication Engineers of Japan.

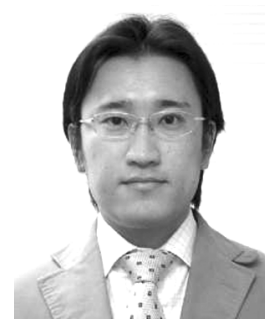

Kunimasa Saitoh (S'00-M'01) was born in Hokkaido, Japan. He received the B.S., M.S., and $\mathrm{Ph} . \mathrm{D}$. degrees in electronic engineering from Hokkaido University, Sapporo, Japan, in 1997, 1999 , and 2001, respectively.

From 1999 to 2001, he was a Research Fellow at Japan Society for the Promotion of Science. From 2001 to 2005, he was a Research Associate at the Graduate School of Engineering, Hokkaido University, where he became an Associate Professor in 2005, and is currently with the Division of Media and Network Technologies. His current research interests include research on fiber optics, nanophotonics, integrated optical devices, and computer-aided design and modeling of guided-wave devices using finite-element method, beam propagation method, etc. He is the author or coauthor of more than 100 research papers in published refereed international journals.

Dr. Saitoh is a Member of the Institute of Electronics, Information and Communication Engineers (IEICE) and the Optical Society of America. In 1999 and 2002, he was awarded the Excellent Paper Award and the Young Scientist Award from the IEICE, respectively, and in 2008, the Young Scientists' Prize of the Commendation for Science and Technology from the Ministry of Education, Culture, Sports, Science, and Technology, Government of Japan.

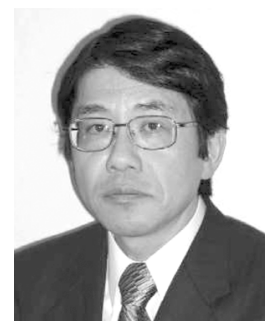

Masanori Koshiba (M'76-SM'87-F'03) was born in Sapporo, Japan. He received the B.S., M.S., and $\mathrm{Ph} . \mathrm{D}$. degrees in electronic engineering from Hokkaido University, Sapporo, Japan, in 1971, 1973 , and 1976, respectively.

In 1976, he joined the Department of Electronic Engineering, Kitami Institute of Technology, Kitami, Japan. From 1979 to 1987, he was an Associate Professor of Electronic Engineering at Hokkaido University, where he became a Professor in 1987, and is currently with the Division of Media and Network Technologies. He has been engaged in research on wave electronics, including microwaves, millimeter-waves, lightwaves, surface acoustic waves, magnetostatic waves, and electron waves, and computer-aided design and modeling of guided-wave devices using finite-element method, boundary element method, beam propagation method, etc. He is the author or coauthor of more than 320 research papers in English and more than 130 research papers in Japanese, both published in refereed journals. He is the author of the books Optical Waveguide Analysis (New York: McGraw-Hill, 1992) and Optical Waveguide Theory by the Finite Element Method (Tokyo, Japan: KTK Scientific/Dordrecht, The Netherlands: Kluwer Academic, 1992), and the coauthor of the books Analysis Methods for Electromagnetic Wave Problems (Boston, MA: Artech House, 1990), Analysis Methods for Electromagnetic Wave Problems, Volume 2 (Boston, MA: Artech House, 1996), Ultrafast and Ultra-parallel Optoelectronics (Chichester, U.K.: Wiley, 1995), and Finite Element Software for Microwave Engineering (New York: Wiley, 1996).

Prof. Koshiba is a Fellow of the Institute of Electronics, Information and Communication Engineers (IEICE), and a Member of the Institute of Electrical Engineers of Japan and the Institute of Image Information and Television Engineers of Japan. In 1987, 1997, and 1999, he was awarded the Excellent Paper Awards from the IEICE, in 1998, the Electronics Award from the IEICE-Electronics Society, and in 2004, the Achievement Award from the IEICE. From 1999 to 2000, he was the President of the IEICE-Electronics Society, and in 2002, he was the Chairman of the IEEE Lasers and Electro-Optics Society Japan Chapter. From 2003 to 2005, he was a member on the Board of Directors of the IEICE. In 2008, he was the Chairman of the IEICE Hokkaido Chapter, and since 2009, he has been the Chairman of the IEEE Sapporo Section. 